\title{
Safety and Efficacy of Prasugrel in Elderly/Low Body Weight Japanese Patients with Ischemic Stroke: Randomized PRASTRO-II
}

\author{
Kazuo Kitagawa ${ }^{a}$ Kazunori Toyodab Takanari Kitazono ${ }^{c}$ Masakatsu Nishikawad \\ Shinsuke Nanto ${ }^{\text {e }}$ Yasuo Ikeda ${ }^{f}$ Kenji Abe ${ }^{g}$ Akira Ogawah $^{\text {h }}$ \\ ${ }^{a}$ Department of Neurology, Tokyo Women's Medical University School of Medicine, Tokyo, Japan; ${ }^{b}$ Department of \\ Cerebrovascular Medicine, National Cerebral and Cardiovascular Center, Osaka, Japan; ' Department of Medicine and

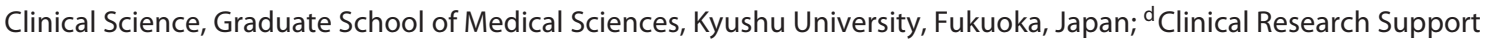 \\ Center, Mie University Hospital, Mie, Japan; ' Department of Cardiology, Nishinomiya Municipal Central Hospital, \\ Hyogo, Japan; fWaseda University Faculty of Science and Engineering, Tokyo, Japan; 9 Daiichi Sankyo Co., Ltd., Tokyo, \\ Japan; ' Department of Neurosurgery, Iwate Medical University, Iwate, Japan
}

\section{Keywords}

Elderly · Body weight · Safety · Therapy · Prasugrel

\begin{abstract}
Introduction: The safety of prasugrel in elderly and/or low body weight Japanese patients with ischemic stroke who have a relatively high bleeding risk with antiplatelet therapy remains unknown. Objective: We aimed to investigate the safety and efficacy of long-term prasugrel monotherapy for stroke prevention compared with clopidogrel in elderly and/ or low body weight Japanese patients with non-cardioembolic ischemic stroke. Methods: In this randomized, doubleblind, comparative, phase III study, elderly (age $\geq 75$ years) and/or low body weight ( $\leq 50 \mathrm{~kg}$ ) Japanese patients with a previous history of non-cardioembolic ischemic stroke were assigned to a prasugrel $3.75 \mathrm{mg}$ (PRA3.75) group, a prasugrel $2.5 \mathrm{mg}$ (PRA2.5) group, or a clopidogrel $50 \mathrm{mg}$ (CLO50) group and followed up for 48 weeks. The primary safety endpoint was the combined incidence of primary safety events, defined as life-threatening, major, and other clinically relevant bleeding. The efficacy endpoint was a composite of ischemic stroke, myocardial infarction, and death from other vascular causes. Results: A total of 654 patients (age $76.4 \pm 7.3$ years, body weight $55.6 \pm 9.3 \mathrm{~kg}$, women $43.9 \%$ ) from 74 medical institu-
\end{abstract}

tions within Japan were enrolled. The combined incidence $(95 \% \mathrm{Cl})$ of primary safety events was $4.2 \%(1.9-7.8 \%), 1.9 \%$ $(0.5-4.7 \%)$, and $3.6 \%(1.6-6.9 \%)$ in the PRA3.75 group ( $n=$ $216)$, PRA2.5 group $(n=215)$, and CLO50 group $(n=223)$, respectively (hazard ratios [HR] PRA3.75/CLO50, 1.13 [0.442.93]; PRA2.5/CLO50, 0.51 [0.15-1.69]). The incidences of bleeding leading to treatment discontinuation $(95 \% \mathrm{CI})$ were $2.3 \%(0.8-5.3 \%), 0.9 \%(0.1-3.3 \%)$, and $2.2 \%(0.7-5.2 \%)$ in the PRA3.75, PRA2.5, and CLO50 groups, respectively (HRs PRA3.75/CLO50, 1.01 [0.29-3.48]; PRA2.5/CLO50, 0.41 [0.082.12]). There was no significant difference in all bleeding events between groups. The incidence of ischemic stroke, myocardial infarction, and death from other vascular causes was lower, but not significantly so, in patients treated with prasugrel than in patients treated with clopidogrel: PRA3.75, 0.0\% (0/216); PRA2.5, 3.3\% (7/215); and CLO50, 3.6\% (8/223; HRs PRA3.75/CLO50, 0.00 [0.00-0.00]; PRA2.5/CLO50, 0.90 [0.32-2.47]). Conclusions: Elderly and/or low body weight Japanese patients with previous non-cardioembolic ischemic stroke who received PRA3.75 showed similar results in terms of primary safety end point, and a numerically lower incidence of ischemic stroke, myocardial infarction, and death from other vascular causes, compared with those who received CLO50.

(c) 2020 The Author(s)

Published by S. Karger AG, Basel

\section{KARGER}

karger@karger.com www.karger.com/ced (c) 2020 The Author(s)

Published by S. Karger AG, Basel

Karger

This article is licensed unde the Cretive Commons AtributionThis article is licensed under the Creative Commons AttributionNonCommercial-NoDerivatives 4.0 International License (CC BY NC-ND) (http://www.karger.com/Services/OpenAccessLicense). Usage and distribution for commercial purposes as well as any distribution of modified material requires written permission.
Kazuo Kitagawa, MD

Department of Neurology

Tokyo Women's Medical University School of Medicine

8-1 Kawada, Shinjuku-ku, Tokyo 162-8666 (Japan)

E-Mail kitagawa.kazuo@ twmu.ac.jp 


\section{Introduction}

Guidelines for management of stroke recommend antiplatelet therapy for secondary prevention of noncardioembolic ischemic stroke, and accordingly, several antiplatelet agents including clopidogrel and aspirin are currently in use [1-3]. However, there are poor responders to aspirin and clopidogrel [4-6], who have high risk of major adverse cardiovascular events [7-10]. Approximately 19\% of Japanese patients are CYP2C19 poor metabolizers (PM) who can only attain a low concentration of the active metabolite of clopidogrel [11]. Thus, it is expected that platelet inhibition with clopidogrel monotherapy would be insufficient in these patients. Compared with clopidogrel, prasugrel has been shown to exhibit stronger platelet aggregation inhibition and to be less susceptible to the effects of CYP2C19 genetic polymorphisms [6-11]. Thus, prasugrel, which shows consistent efficacy in CYP2C19 PMs, would be a better option for stroke prevention in Japanese patients.

The PRASTRO-I study (a pivotal phase III study) investigated the noninferiority of prasugrel $3.75 \mathrm{mg}$ (PRA3.75) to clopidogrel $75 \mathrm{mg}$ (CLO75) for stroke prevention in non-cardioembolic ischemic stroke patients. A total of 3,747 patients were enrolled. In accordance with the clopidogrel package insert [12], elderly (age $\geq 75$ years) and low body weight $(\leq 50 \mathrm{~kg})$ patients were excluded [13]. Thus, we conducted the present study (PRASTROII: Safety and efficacy of PRAsugrel in elderly and/or low body weight Japanese patients with ischemic STROke) to investigate the safety and efficacy of prasugrel in elderly and/or low body weight Japanese patients with a history of non-cardioembolic ischemic stroke. The doses of prasugrel (3.75 and $2.5 \mathrm{mg}$ ) in the present study were based on the results of a previous clinical pharmacology study [14].

\section{Patients and Methods}

\section{Study Design}

This was a multicenter, randomized, double-blind, 3-group, comparative study of elderly (age $\geq 75$ years) and/or low body weight $(\leq 50 \mathrm{~kg})$ Japanese patients with a history of non-cardioembolic ischemic stroke. Thus, there was no upper limit for the body weight of patients aged $\geq 75$ years, but patients aged $<75$ years were eligible only if their body weight was $\leq 50 \mathrm{~kg}$. Patients were stratified by age and body weight, and then randomly assigned to a prasugrel $3.75 \mathrm{mg}$ (PRA3.75), prasugrel $2.5 \mathrm{mg}$ (PRA2.5), or clopidogrel $50 \mathrm{mg}$ (CLO50) group, in a 1:1:1 ratio. The randomization schedule was generated by an independent statistician, using SAS software (version 9.2) to create a computer-generated random number sequence. A double-dummy design ensured that patients, investigators, and the funding organization were masked to treatment allocation. Patients were followed up for $>48$ weeks. The dose of $50 \mathrm{mg}$ for clopidogrel was determined based on the dosage indication for clopidogrel in Japan [12]. The study was conducted at 74 medical institutions within Japan from September 2012 to October 2014.

The target sample size was 600 patients from among patients with ischemic stroke. The target number of patients in each group was determined to be 200 , in whom primary safety events, defined as all life-threatening, major, and other clinically relevant bleeding, would be expected to occur at a frequency of $\geq 1.5 \%$ with a probability of $95 \%$.

Elderly patients (age $\geq 75$ years) and/or low body weight $(\leq 50 \mathrm{~kg})$ patients were eligible if they had non-cardioembolic ischemic stroke ( $\geq 4$ weeks before giving consent). Eligible patients had an infarct area, considered to be the cause of the last attack, confirmed on brain MRI, and were classified according to stroke types in the TOAST classification [15]. Patients were excluded if they met any of the following criteria: presence of cardioembolic stroke, paradoxical embolism, or silent brain infarction; coadministration of other antiplatelet agents; current evidence or previous history of cerebral hemorrhage, high risk of subarachnoid hemorrhage, or poorly controlled hypertension; and body weight $<40 \mathrm{~kg}$.

Patients in each group received a combination of the study drug taken orally once daily after breakfast for 48 weeks. During the study, blood pressure was controlled (target level, 140/90 mm Hg). Concomitant use of antiplatelet agents, anticoagulants, thrombolytic agents, acidic nonsteroidal anti-inflammatory drugs, and other study drugs was prohibited throughout the study period. An independent data-monitoring committee was established to review data.

\section{Endpoints}

Bleeding events and efficacy events were reviewed by the bleeding event committee and the efficacy event committee, respectively, under blinded conditions.

\section{Safety}

The primary endpoint was the combined incidence of primary safety events, defined as life-threatening bleeding, major bleeding, and other clinically relevant bleeding. We also evaluated cumulative event incidences in each group. We performed stratified analyses according to age and body weight ( $\geq 75$ years and $>50 \mathrm{~kg},<75$ years and $\leq 50 \mathrm{~kg}, \geq 75$ years and $\leq 50 \mathrm{~kg}$ ). As secondary endpoints, incidences of individual types of bleeding event were evaluated.

The definitions of the bleeding events are given in the online Supplemental Methods (for all online material, see www.karger. com/doi/10.1159/000506825). Adverse events (AE) observed during the study period were recorded.

\section{Efficacy}

The efficacy endpoints were the incidence of a composite of ischemic stroke, myocardial infarction, and death from other vascular causes; ischemic cerebrovascular events (ischemic stroke and transient ischemic attack); and any stroke. Definitions of the efficacy events are provided in the online Supplemental Methods. Platelet function was assessed using the VerifyNow ${ }^{\circledR}$ assay (Accriva Diagnostics, Inc., San Diego, CA, USA). This measures adenosine diphosphate-induced platelet function 
in terms of increase in light transmittance and yields values in $\mathrm{P} 2 \mathrm{Y} 12$ reaction units (PRU) [16]. The assay was performed before the start of treatment and at 4, 24, and 48 weeks after treatment. Details of genotyping are provided in the online Supplemental Methods.

\section{Statistical Analysis}

The safety analysis was performed on the safety analysis set, defined as all randomized patients, excluding those with serious Good Clinical Practice (GCP) violations and those who had not taken the study drug even once. The efficacy analysis was performed on the full analysis set (FAS), defined as all randomized patients, excluding those with serious GCP violations, those who had not taken the study drug even once, and those for whom there were no data after taking the study drug. The pharmacodynamic analysis set comprised patients from the FAS who had adequate pharmacodynamic data to derive at least one pharmacodynamic measurement after administration of the study drug.

For the primary endpoint and efficacy endpoints, event incidences and 95\% CIs were calculated for each treatment group. Additionally, hazard ratios and 95\% CIs of the PRA3.75 and PRA2.5 groups against the CLO50 group were calculated. Cumulative incidences in each group were estimated by the Kaplan-Meier method and presented graphically. Summary statistics for PRU measurements at each time point were calculated, and differences in the mean values between the PRA groups and the CLO50 group and their 95\% CIs were calculated. The same calculations were made for each genotype.

\section{Trial Registration}

The study was preregistered with the Japan Pharmaceutical Information Center (http://www.clinicaltrials.jp/user/showCteDetailE.jsp?japicId = JapicCTI-121901, JapicCTI-121901).

\section{Results}

\section{Patients}

The progress of patients in the trial is shown in online Supplemental Figure 1. All 654 patients were confirmed to have taken the study drug at least once and were included in the safety analysis set and FAS.

Table 1 shows the baseline characteristics of each group. The greatest proportion $(62.4 \%)$ comprised patients aged $\geq 75$ years with body weight $>50 \mathrm{~kg}$, followed by those aged $<75$ years with body weight $\leq 50 \mathrm{~kg}(20.2 \%)$, and those aged $\geq 75$ years with body weight $\leq 50 \mathrm{~kg}(17.4 \%)$. Regarding the types of stroke, small artery occlusion (lacunae) was the most frequent (approximately 35\% of strokes). Large artery atherosclerosis and stroke of undetermined etiology accounted for approximately 30\% each. As for CYP2C19 phenotypes, PMs accounted for $17.6 \%$. In terms of baseline characteristics, there were no differences between groups.

\section{Safety}

Primary Endpoint

Table 2 shows the incidences of primary safety events. Figure 1a shows the cumulative incidence of primary safety events in each group.

The combined incidence of primary safety events (i.e., the primary endpoint) was $4.2,1.9$, and $3.6 \%$ in the PRA3.75, PRA2.5, and CLO50 groups, respectively. Hazard ratios $(95 \% \mathrm{CI})$ for primary safety events in the PRA3.75 group and PRA2.5 group relative to the CLO50 group were $1.13(0.44-2.93)$ and $0.51(0.15-$ 1.69), respectively. Among bleeding events recorded as primary safety events, life-threatening bleeding occurred in 3 patients (1 patient each with subdural hematoma, hemorrhagic gastric ulcer, and subarachnoid hemorrhage) in the PRA3.75 group, 1 patient (cardiac tamponade) in the PRA2.5 group, and none in the CLO50 group.

Intracerebral hemorrhage did not occur in any of the groups. Online Supplemental Table 1 shows the primary endpoint results stratified by patient age and body weight.

\section{Secondary Endpoints}

Table 2 shows the results for the secondary endpoints, defined as the incidences of individual types of bleeding event. The incidences of all bleeding events were 31.9, 24.7, and 23.3\% in the PRA3.75, PRA2.5, and CLO50 groups, respectively. However, there was no significant difference in all bleeding events between groups.

\section{Adverse Events}

Incidences of AEs did not differ between groups (online Supplemental Table 2). No deaths were considered causally related to the study drugs. The incidence of drug-related serious AEs did not differ between groups. Incidences of drug-related AEs leading to discontinuation were $2.8,3.7$, and $6.7 \%$ in the PRA3.75, PRA2.5, and CLO50 groups, respectively.

\section{Efficacy}

Table 3 shows the incidences of the efficacy events, and Figure $1 \mathrm{~b}$ shows the cumulative incidence of ischemic stroke, myocardial infarction, and death from other vascular causes in each group. None of the patients who received $3.75 \mathrm{mg}$ prasugrel had ischemic stroke or myocardial infarction, or death from other vascular causes defined in this study. The results for all efficacy endpoints were numerically lower in the PRA3.75 group than in the PRA2.5 and CLO50 groups. 
Table 1. Patient characteristics ${ }^{\mathrm{a}}$

\begin{tabular}{|c|c|c|c|}
\hline & PRA3.75 $(n=216)$ & PRA2.5 $(n=215)$ & $\operatorname{CLO} 50(n=223)$ \\
\hline Age, years, mean \pm SD & $76.1 \pm 7.6$ & $76.7 \pm 7.0$ & $76.4 \pm 7.3$ \\
\hline Body weight, kg, mean \pm SD & $55.0 \pm 8.9$ & $55.9 \pm 9.1$ & $56.0 \pm 9.7$ \\
\hline \multicolumn{4}{|l|}{ Age and body weight, $n(\%)$} \\
\hline$\geq 75$ years and $>50 \mathrm{~kg}$ & $131(60.6)$ & $141(65.6)$ & $136(61)$ \\
\hline$<75$ years and $\leq 50 \mathrm{~kg}$ & $44(20.4)$ & $36(16.7)$ & $52(23.3)$ \\
\hline$\geq 75$ years and $\leq 50 \mathrm{~kg}$ & $41(19)$ & $38(17.7)$ & $35(15.7)$ \\
\hline Women, $n(\%)$ & $97(44.9)$ & $94(43.7)$ & $96(43)$ \\
\hline \multicolumn{4}{|l|}{ Smoking, $n(\%)$} \\
\hline Never smoker & $98(45.4)$ & $103(47.9)$ & $99(44.4)$ \\
\hline Previous smoker & $107(49.5)$ & $93(43.3)$ & $105(47.1)$ \\
\hline Current smoker & $11(5.1)$ & $19(8.8)$ & $19(8.5)$ \\
\hline \multicolumn{4}{|l|}{ Blood pressure, $\mathrm{mm} \mathrm{Hg}$, mean $\pm \mathrm{SD}$} \\
\hline Systolic blood pressure & $132.6 \pm 14.4$ & $133.6 \pm 14.9$ & $132.6 \pm 14.2$ \\
\hline Diastolic blood pressure & $74.3 \pm 10.2$ & $73.5 \pm 10.1$ & $73.3 \pm 12.1$ \\
\hline Hemoglobin, g/dL, mean \pm SD & $13.3 \pm 1.4$ & $13.3 \pm 1.3$ & $13.3 \pm 1.3$ \\
\hline \multicolumn{4}{|l|}{ Low-density lipoprotein cholesterol, } \\
\hline Creatinine, $\mathrm{mg} / \mathrm{dL}$, mean $\pm \mathrm{SD}$ & $0.788 \pm 0.241$ & $0.784 \pm 0.217$ & $0.764 \pm 0.195$ \\
\hline \multicolumn{4}{|c|}{ Duration from last stroke attack to treatment start, weeks, $n(\%)$} \\
\hline$\geq 4,<12$ & $51(23.6)$ & $59(27.4)$ & $61(27.4)$ \\
\hline$\geq 12,<26$ & $36(16.7)$ & $25(11.6)$ & $27(12.1)$ \\
\hline$\geq 26,<52$ & $23(10.6)$ & $19(8.8)$ & $21(9.4)$ \\
\hline$\geq 52$ & $106(49.1)$ & $112(52.1)$ & $114(51.1)$ \\
\hline \multicolumn{4}{|l|}{ Type of last stroke, $n(\%)$} \\
\hline Large artery atherosclerosis & $64(29.6)$ & $70(32.6)$ & $78(35.0)$ \\
\hline Small artery occlusion & $82(38.0)$ & $84(39.1)$ & $69(30.9)$ \\
\hline Stroke of other etiology & $1(0.5)$ & $3(1.4)$ & $2(0.9)$ \\
\hline Stroke of undetermined etiology & $69(31.9)$ & $58(27.0)$ & $74(33.2)$ \\
\hline \multicolumn{4}{|l|}{ Modified Rankin Scale, $n(\%)$} \\
\hline Grade 0 & $52(24.1)$ & $63(29.3)$ & $72(32.3)$ \\
\hline Grade 1 & $113(52.3)$ & $108(50.2)$ & $106(47.5)$ \\
\hline Grade 2 & $31(14.4)$ & $26(12.1)$ & $32(14.3)$ \\
\hline Grade 3 & $13(6.0)$ & $17(7.9)$ & $10(4.5)$ \\
\hline Grade 4 & $7(3.2)$ & $1(0.5)$ & $3(1.3)$ \\
\hline \multicolumn{4}{|l|}{ History of arteriosclerosis, $n(\%)$} \\
\hline Ischemic stroke & $30(13.9)$ & $36(16.7)$ & $29(13.0)$ \\
\hline Transient ischemic attack & $16(7.4)$ & $16(7.4)$ & $13(5.8)$ \\
\hline Acute myocardial infarction & $0(0.0)$ & $1(0.5)$ & $1(0.4)$ \\
\hline Unstable angina & $1(0.5)$ & $4(1.9)$ & $2(0.9)$ \\
\hline Chronic arteriosclerosis obliterans & $5(2.3)$ & $2(0.9)$ & $5(2.2)$ \\
\hline \multicolumn{4}{|l|}{ Complication, $n(\%)$} \\
\hline Hypertension & $177(81.9)$ & $176(81.9)$ & $175(78.5)$ \\
\hline Dyslipidemia & $141(65.3)$ & $121(56.3)$ & $131(58.7)$ \\
\hline Diabetes mellitus & $66(30.6)$ & $71(33.0)$ & $66(29.6)$ \\
\hline \multicolumn{4}{|l|}{ CYP2C19 phenotype, $n(\%)$} \\
\hline Extensive metabolizer & $66(30.6)$ & $58(27.0)$ & $62(27.8)$ \\
\hline Intermediate metabolizer & $94(43.5)$ & $106(49.3)$ & $97(43.5)$ \\
\hline Poor metabolizer & $36(16.7)$ & $31(14.4)$ & $36(16.1)$ \\
\hline Missing & $20(9.3)$ & $20(9.3)$ & $28(12.6)$ \\
\hline
\end{tabular}

a No significant difference was found for any of the comparisons.

PRA3.75, prasugrel 3.75 mg; PRA2.5, prasugrel $2.5 \mathrm{mg}$; CLO50, clopidogrel $50 \mathrm{mg}$. 
Table 2. Incidences of bleeding events ${ }^{\mathrm{a}}$

Number of patients, incidence (95\% CI)

\begin{tabular}{|c|c|c|c|c|}
\hline PRA3.75 $(n=216)$ & PRA2.5 $(n=215)$ & CLO50 $(n=223)$ & PRA3.75/CLO50 & PRA2.5/CLO50 \\
\hline$n$ & $n \quad \%$ & $n \quad \%$ & & \\
\hline
\end{tabular}

Primary endpoint

Primary safety events

Life-threatening bleeding

Major bleeding

Other clinically relevant bleeding

\begin{tabular}{rcrcrccc}
9 & $4.2(1.9-7.8)$ & 4 & $1.9(0.5-4.7)$ & 8 & $3.6(1.6-6.9)$ & $1.13(0.44-2.93)$ & $0.51(0.15-1.69)$ \\
3 & $1.4(0.3-4.0)$ & 1 & $0.5(0.0-2.6)$ & 0 & $0.0(0.0-1.6)$ & - & - \\
0 & $0.0(0.0-1.7)$ & 1 & $0.5(0.0-2.6)$ & 0 & $0.0(0.0-1.6)$ & - & - \\
6 & $2.8(1.0-5.9)$ & 2 & $0.9(0.1-3.3)$ & 8 & $3.6(1.6-6.9)$ & $0.76(0.26-2.18)$ & $0.26(0.05-1.20)$ \\
\hline & & & & & & & \\
5 & $2.3(0.8-5.3)$ & 2 & $0.9(0.1-3.3)$ & 5 & $2.2(0.7-5.2)$ & $1.01(0.29-3.48)$ & $0.41(0.08-2.12)$ \\
69 & $31.9(25.8-38.6)$ & 53 & $24.7(19.0-31.0)$ & 52 & $23.3(17.9-29.4)$ & $1.40(0.98-2.00)$ & $1.04(0.71-1.53)$
\end{tabular}

Secondary endpoints

Bleeding leading to treatment discontinuation

All bleeding events

a No significant difference was found for any of the comparisons.

$\mathrm{HR}$, hazard ratio; PRA3.75, prasugrel $3.75 \mathrm{mg}$; PRA2.5, prasugrel $2.5 \mathrm{mg}$; CLO50, clopidogrel $50 \mathrm{mg}$.

Fig. 1. Cumulative incidences of primary safety events (a) and ischemic stroke, myocardial infarction, and death from other vascular causes (b). The vertical lines indicate censored data. PRA3.75, prasugrel $3.75 \mathrm{mg}$; PRA2.5, prasugrel $2.5 \mathrm{mg}$; CLO50, clopidogrel $50 \mathrm{mg}$.

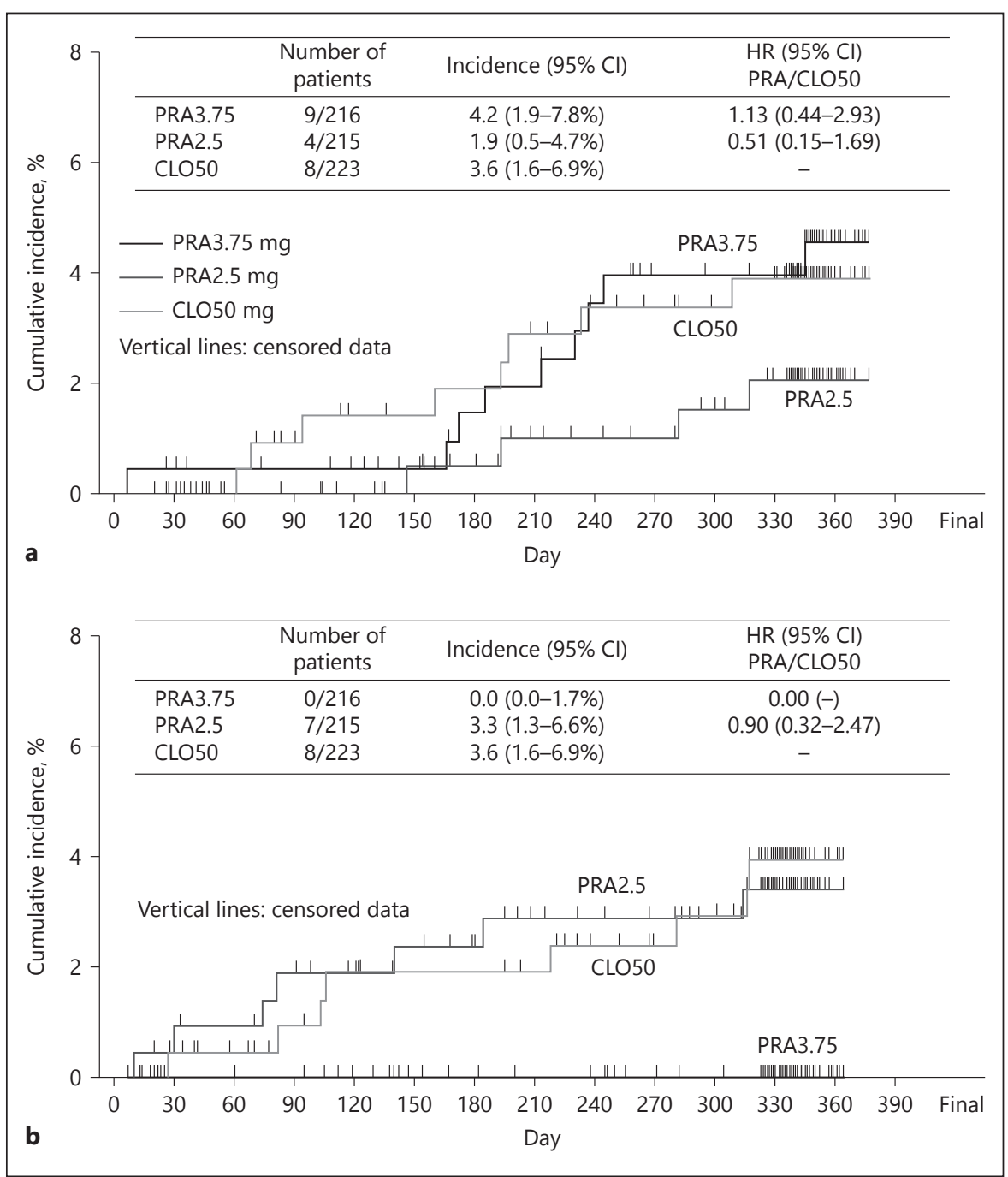


Table 3. Efficacy events ${ }^{a}$

Number of patients, incidence (95\% CI)

PRA3.75 $(n=216) \quad$ PRA2.5 $(n=215)$

n $\%$
RA2.5 $(n=215)$

$\%$ n

$0 / 216 \quad 0.0(0.0-1.7)$

$0 / 216 \quad 0.0(0.0-1.7) \quad 7 / 215$

$0 / 216 \quad 0.0(0.0-1.7)$

$0 / 216 \quad 0.0(0.0-1.7)$

$0 / 216 \quad 0.0(0.0-1.7)$

$1 / 216 \quad 0.5(0.0-2.6)$
HR (95\% CI)

PRA3.75/CLO50 PRA2.5/CLO50

CLO50 $(n=223)$

$\%$ infarction, and death from other vascular causes

Myocardial infarction

Death from other vascular causes

Any stroke

$7 / 215$
$7 / 215$
$0 / 215$
$0 / 215$
$7 / 215$

$3.3(1.3-6.6)$

$3.3(1.3-6.6)-8 / 223$

$3.3(1.3-6.6)$
$0.0(0.0-1.7)$

$0.0(0.0-1.7)$

$3.7(1.6-7.2)$

$3.3(1.3-6.6)$
$8 / 223$
$6 / 223$
$2 / 223$
$0 / 223$
$6 / 223$
$6 / 223$

$3.6(1.6-6.9)$

$2.7(1.0-5.8)$

$0.9(0.1-3.2)$

$0.0(0.0-1.6)$

$2.7(1.0-5.8)$

$2.7(1.0-5.8)$
$0.00(-)$

$0.00(-)$

$0.00(-)$

$0.00(-)$

$0.17(0.02-1.37)$
$0.90(0.32-2.47)$

$1.19(0.40-3.55)$

$0.00(-)$

$1.36(0.47-3.93)$

$1.19(0.40-3.55)$

${ }^{a}$ No significant difference was found for any of the comparisons

$\mathrm{HR}$, hazard ratio; PRA3.75, prasugrel $3.75 \mathrm{mg}$; PRA2.5, prasugrel $2.5 \mathrm{mg}$; CLO50, clopidogrel $50 \mathrm{mg}$.

\section{Pharmacodynamics}

Online Supplemental Figure 2a shows changes in PRU values. Mean PRU values in the PRA3.75, PRA2.5, and CLO50 groups were 139.0, 196.6, and 223.2, respectively, at week 4 .

Online Supplemental Figure $2 \mathrm{~b}$ shows PRU values at week 4 according to CYP2CI9 phenotype. In the PRA3.75 and PRA2.5 groups, mean PRU values were similarly low, irrespective of CYP2C19 phenotype. In the CLO50 group, mean PRU value was lowest in EMs, followed by IMs and PMs, reflecting the influence of CYP2C19 genetic polymorphisms.

\section{Discussion}

Compared with the CLO50 group, the combined incidence of primary safety events (the primary endpoint) was similar in the PRA3.75 group and lower (but nonsignificantly so) in the PRA2.5 group. The findings suggest that PRA3.75 can be used effectively and safely in highrisk patients such as elderly patients and those with low body weight. In the PRA3.75 group, the incidence of the primary safety events was at a similar level to that in the CLO50 group. In the analysis stratified by age and body weight, no such event was observed in the subgroup of patients aged $\geq 75$ years with body weight $\leq 50 \mathrm{~kg}$, which is considered at a greatest risk of bleeding. Furthermore, no efficacy events were observed in this group. The absence of events in these higher risk patients is noteworthy; however, because of the small number of patients in this subgroup, it was not possible to establish statistical significance. The PRA2.5 group showed numerically (but not significantly) lower incidence of the primary safety events, and similar incidence of the efficacy events, to the CLO50 group. Therefore, we consider prasugrel at this dose to be not numerically inferior to CLO50 in terms of safety and efficacy in elderly and/or low body weight patients.

Although this study lacked the statistical power to detect vascular events, our data showing no ischemic events with prasugrel $3.75 \mathrm{mg}$ in approximately $200 \mathrm{pa}-$ tients in a 1-year period suggest that high and potent inhibition of P2Y12 may reduce ischemic stroke, myocardial infarction, and death from other vascular causes in patients with ischemic stroke; this finding is consistent with that shown in ACS patients undergoing PCI in previous studies [17]. Two regimens with similar inhibition of P2Y12, CLO50, and PRA2.5 showed similar incidences of several efficacy endpoints. Moreover, the PRU values in patients who received treatment with prasugrel were consistently low, irrespective of CYP2C19 genetic polymorphisms. However, the clinical question of superior efficacy of prasugrel over clopidogrel in stroke prevention requires a larger clinical event study.

\section{Conclusion}

In elderly and/or low body weight Japanese patients with non-cardioembolic ischemic stroke, prasugrel (3.75 or $2.5 \mathrm{mg}$ ) showed similar results in terms of combined incidence of primary safety events, compared with CLO50. Regarding efficacy for the prevention of ischemic stroke, myocardial infarction, and death from other vascular causes, PRA3.75 resulted in a numerically lower incidence compared with CLO50. 


\section{Acknowledgments}

The authors deeply appreciate the contributions of all the investigators and other clinical/research staff involved in the present study. The authors also wish to thank Tetsuhiko Yokoyama and Rie Ishibashi, on behalf of inScience Communications, Springer Healthcare, for medical writing support; this was funded by Daiichi Sankyo Co., Ltd.

\section{Statement of Ethics}

The study fully adheres to the ethical principles of the Declaration of Helsinki as well as GCP guidelines. The study protocol was approved by the Ethics Committee of each participating institution. All study participants provided written informed consent.

\section{Disclosure Statement}

K.K. reports grants and personal fees from Takeda Pharmaceutical, Nippon Boehringer Ingelheim, Sumitomo Dainippon Pharma, Astellas Pharma, Kyowa Hakko Kirin, Otsuka Pharmaceutical, Bayer Yakuhin, and Sanofi; personal fees from Daiichi Sankyo, Mitsubishi Tanabe Pharma, Shionogi, Pfizer, and Bristol-Myers Squibb; and grants from AstraZeneca and Eisai outside the submitted work; K.T. reports personal fees from Daiichi Sankyo, Bayer Yakuhin, Bristol-Myers Squibb, and Nippon Boehringer Ingelheim outside the submitted work; T.K. reports grants and personal fees from Daiichi Sankyo; personal fees from Bayer Yakuhin; and grants from Sanofi, Takeda Pharmaceutical, Chugai Pharmaceutical, Astellas Pharma, Nippon Boehringer Ingelheim, MSD, BristolMyers Squibb, EA Pharma, Shionogi, Mitsubishi Tanabe Pharma, Eisai, Pfizer, Torii Pharmaceutical, Otsuka Pharmaceutical, and Asahi Kasei Medical, outside the submitted work; M.N. reports grants and personal fees from Daiichi Sankyo, personal fees from Sanofi during the conduct of the study; S.N., K.A., and A.O. reports personal fees from Daiichi Sankyo outside the submitted work; Y.I. reports grants from Daiichi Sankyo outside the submitted work.

\section{Funding Sources}

This study and article processing charge were funded by Daiichi Sankyo Co., Ltd. (Tokyo, Japan).

\section{Author Contributions}

K.K., K.T., T.K., M.N., and S.N. contributed to interpretation of data and writing the draft of the manuscript; Y.I. and A.O. contributed to conception or design of the work, interpretation of data, and writing the draft of the manuscript; and K.A. contributed to conception or design of the work, analysis and interpretation of data, and writing the draft of the manuscript. All authors critically reviewed the manuscript, approved the final version of the manuscript, and agree to be accountable for all aspects of the work in ensuring that questions related to the accuracy or integrity of any part of the work are appropriately investigated and resolved.

\section{Data Sharing}

Deidentified individual participant data and relevant supporting clinical trial documents are available on request at https:// www.clinicalstudydatarequest.com//. In cases in which clinical trial data and supporting documents are provided pursuant to Daiichi Sankyo company policies and procedures, Daiichi Sankyo will continue to protect the privacy of our clinical trial participants. Details of data-sharing criteria and the procedure for requesting access can be found at https://vivli.org/ourmember/daiichi-sankyo/. Supporting information includes the study protocol, statistical analysis plan and clinical study report. The access criteria are defined as follows: formal request from qualified scientific and medical researchers on individual participant data and clinical study documents from clinical trials supporting products submitted and licensed in the United States, the European Union, and/or Japan from January 1, 2014 and beyond for the purpose of conducting legitimate research; this must be consistent with the principle of safeguarding study participants' privacy and consistent with provision of informed consent.

\section{References}

1 Japan Stroke Society Committee on Guidelines for the Management of Stroke. Japanese Guidelines for the Management of Stroke, updated version 2017. Tokyo: Kyowa Kikaku; 2017. pp. 43-54.

2 Kernan WN, Ovbiagele B, Black HR, Bravata DM, Chimowitz MI, Ezekowitz MD, et al.; American Heart Association Stroke Council, Council on Cardiovascular and Stroke Nursing, Council on Clinical Cardiology, and Council on Peripheral Vascular Disease. Guidelines for the prevention of stroke in patients with stroke and transient ischemic attack: a guideline for healthcare professionals from the American Heart Association/American Stroke Association. Stroke. 2014 Jul;45(7):2160-236.
3 Piepoli MF, Hoes AW, Agewall S, Albus C, Brotons C, Catapano AL, et al.; ESC Scientific Document Group. 2016 European Guidelines on cardiovascular disease prevention in clinical practice: The Sixth Joint Task Force of the European Society of Cardiology and Other Societies on Cardiovascular Disease Prevention in Clinical Practice (constituted by representatives of 10 societies and by invited experts)Developed with the special contribution of the European Association for Cardiovascular Prevention \& Rehabilitation (EACPR). Eur Heart J. 2016 Aug;37(29): 2315-81.

4 Liu XF, Cao J, Fan L, Liu L, Li J, Hu GL, et al. Prevalence of and risk factors for aspirin re- sistance in elderly patients with coronary artery disease. J Geriatr Cardiol. 2013 Mar; 10(1):21-7.

5 Meves SH, Hummel T, Endres HG, Mayböck N, Kaiser AF, Schröder KD, et al. Effectiveness of antiplatelet therapy in atherosclerotic disease: comparing the ASA lowresponse prevalence in $\mathrm{CVD}, \mathrm{CAD}$ and PAD. J Thromb Thrombolysis. 2014;37(2): 190-201.

6 Liu R, Zhou ZY, Chen YB, Li JL, Yu WB, Chen $\mathrm{XM}$, et al. Associations of CYP3A4, NR1I2, CYP2C19 and P2RY12 polymorphisms with clopidogrel resistance in Chinese patients with ischemic stroke. Acta Pharmacol Sin. 2016 Jul;37(7):882-8. 
7 Yi X, Wang C, Liu P, Fu C, Lin J, Chen Y. Antiplatelet drug resistance is associated with early neurological deterioration in acute minor ischemic stroke in the Chinese population. J Neurol. 2016 Aug;263(8): 1612-9.

8 Feher G, Feher A, Pusch G, Koltai K, Tibold A, Gasztonyi B, et al. Clinical importance of aspirin and clopidogrel resistance. World J Cardiol. 2010 Jul;2(7):171-86.

9 Gengo FM, Rainka M, Robson M, Gengo MF, Forrest A, Hourihane M, et al. Prevalence of platelet nonresponsiveness to aspirin in patients treated for secondary stroke prophylaxis and in patients with recurrent ischemic events. J Clin Pharmacol. 2008 Mar;48(3): 335-43.

10 Krasopoulos G, Brister SJ, Beattie WS, Buchanan MR. Aspirin "resistance" and risk of cardiovascular morbidity: systematic review and meta-analysis. BMJ. 2008 Jan;336(7637): 195-8.

11 Yamamoto K, Hokimoto S, Chitose T, Morita K, Ono T, Kaikita K, et al. Impact of CYP2C19 polymorphism on residual platelet reactivity in patients with coronary heart disease during antiplatelet therapy. J Cardiol. 2011 Mar; 57(2):194-201.

12 Plavix ${ }^{\circledR} 25 \mathrm{mg} / 75 \mathrm{mg}$. Package insert (version 20; last updated in March 2018). Tokyo: Sanofi K.K. (In Japanese).

13 Ogawa A, Toyoda K, Kitagawa K, Kitazono T, Nagao T, Yamagami H, et al.; PRASTRO-I Study Group. Comparison of prasugrel and clopidogrel in patients with non-cardioembolic ischaemic stroke: a phase 3, randomised, non-inferiority trial (PRASTRO-I). Lancet Neurol. 2019 Mar;18(3):238-47.

14 Kitazono T, Ikeda Y, Nishikawa M, Yoshiba $\mathrm{S}$, Abe K, Ogawa A. Influence of cytochrome
P450 polymorphisms on the antiplatelet effects of prasugrel in patients with non-cardioembolic stroke previously treated with clopidogrel. J Thromb Thrombolysis. 2018 Nov; 46(4):488-95.

15 Adams HP Jr, Bendixen BH, Kappelle LJ, Biller J, Love BB, Gordon DL, et al. Classification of subtype of acute ischemic stroke. Definitions for use in a multicenter clinical trial. TOAST. Trial of Org 10172 in Acute Stroke Treatment. Stroke. 1993 Jan;24(1):35-41.

16 Price MJ. Bedside evaluation of thienopyridine antiplatelet therapy. Circulation. 2009 May;119(19):2625-32.

17 Saito S, Isshiki T, Kimura T, Ogawa H, Yokoi $\mathrm{H}$, Nanto S, et al. Efficacy and safety of adjusted-dose prasugrel compared with clopidogrel in Japanese patients with acute coronary syndrome: the PRASFIT-ACS study. Circ J. 2014; 78(7):1684-92. 